\title{
Ocorrência simultânea de COVID-19 e dengue: o que os dados revelam?
}

\author{
Simultaneous occurrence of COVID-19 and \\ dengue: what do the data show?
}

\author{
Ocurrencia simultánea de COVID-19 y dengue: \\ ¿qué revelan los datos?
}

\author{
Márcio Dênis Medeiros Mascarenhas 1 \\ Francisca Miriane de Araújo Batista 2 \\ Malvina Thais Pacheco Rodrigues 1 \\ Ocimar de Alencar Alves Barbosa 3 \\ Veruska Cavalcanti Barros 1
}

doi: 10.1590/0102-311X00126520
A COVID-19, doença emergente causada pelo novo coronavírus denominado SARS-CoV-2, tem provocado impactos em todos os setores da sociedade, principalmente nos sistemas de saúde, devido à sua rápida disseminação por todos os continentes, sua capacidade de provocar mortes em populações vulneráveis, além do insuficiente conhecimento científico sobre o vírus, patogenia e tratamento. Em 14 de maio de 2020, o número de casos confirmados em nível global era de 4.307.287 com 295.101 mortes $1,2,3,4$.

No Brasil, as condições precárias de habitação e saneamento caracterizadas por aglomeração e ausência de acesso constante à água tratada, os desfavoráveis indicadores socioeconômicos que refletem a qualidade de vida da maioria da população, juntamente com a alta prevalência de doenças crônicas, como hipertensão e diabetes, que são fatores de risco para a COVID-19, ampliam os impactos e dificultam as estratégias de enfrentamento da doença, com a possibilidade iminente de colapso dos serviços de saúde em diversos estados 5 .

Além da epidemia de COVID-19, o Brasil enfrenta epidemias de dengue desde 1986, sazonalmente de março a junho. A elevação no nível pluviométrico e as falhas nas ações de controle do mosquito vetor (Aedes aegypti) contribuem para o aumento de casos de dengue nesse período. Situação semelhante é observada com doenças respiratórias como a influenza, que apresenta surtos sazonais, principalmente no outono e no inverno. Com a coincidência espaço-temporal desses agravos, os serviços do Sistema Único de Saúde (SUS), que já apresentavam deficiências no atendimento para dengue e outras doenças, tiveram de se adaptar bruscamente para ampliar sua estrutura física, adquirir equipamentos e insumos, construir hospitais de campanhas, capacitar os profissionais e aumentar a capacidade de testagem 6,7,8,9,10,11,12. Porém, o impacto da COVID-19 no sistema público de saúde é perceptível pela elevada demanda por internação hospitalar, levando ao esgotamento da oferta de leitos de terapia intensiva e ventiladores pulmonares em algumas regiões do país 13 .

COVID-19 e dengue apresentam semelhanças clínicas e laboratoriais. Yan et al. 14 observaram que pacientes infectados pelo SARS-CoV-2 e diagnosticados com dengue, por meio de teste rápido, evoluíram para quadros clínicos mais graves, retardando o tratamento eficaz. Diagnósticos falsos positivos e métodos laboratoriais menos sensíveis podem gerar, não somente, complicações na saúde do paciente, como favorecem a expansão da COVID-19, sobrecarregando os sistemas de saúde pública $6,14,15$.
1 Universidade Federal do Piaui, Teresina, Brasil. 2 Centro de Inteligência em Agravos Tropicais Emergentes e Negligenciados, Universidade Federal do Piaui, Teresina, Brasil. ${ }^{3}$ Secretaria Estadual de Saúde do Piauí, Teresina, Brasil.

\section{Correspondência}

F. M. A. Batista

Centro de Inteligência em Agravos Tropicais Emergentes e Negligenciados, Universidade Federal do Piauí.

Av. Jornalista Dondon 2883,

Teresina, PI 64052-850,

Brasil.

mirianearaujo@hotmail.com 
O número de notificações de dengue no Brasil até a semana epidemiológica (SE) 17 de 2020 ultrapassava o número de casos observados na SE 7 de 2015 e na SE 11 de 2019. Entretanto, a partir da SE 10 percebeu-se um declínio do número de notificações de dengue, coincidentemente no período em que as ações de saúde do país foram intensificadas para o combate da COVID-19, sugerindo uma possível subnotificação num período em que é esperado o aumento sazonal de casos de dengue no Brasil 16.

Em relação à incidência de casos de dengue no país, a Região Nordeste apresentava a segunda menor incidência da doença quando comparada com as demais regiões do país (82,5/100 mil habitantes) até a SE 17 de 2020. No Estado do Piauí, a incidência acumulada de dengue foi de 20,2/100 mil habitantes até a SE 17 de 2020, demonstrando redução de 74,5\% em relação ao mesmo período de 2019 16,17. Observando a série histórica recente de dengue no estado, percebe-se que a incidência da doença em 2020 estava próxima do padrão de ocorrência demonstrado pela média do coeficiente para os anos de 2016 a 2019 17. No entanto, a partir da semana em que foram registrados os primeiros casos confirmados de COVID-19 no Piauí (SE 12) 18, verificou-se o aumento exponencial de sua incidência simultaneamente à queda da incidência de dengue (Figura 1).

Essa mudança abrupta no comportamento dos dados epidemiológicos da dengue reforça a hipótese de subnotificação dos casos no Piauí. Observando apenas os coeficientes de incidência atualmente disponíveis, estima-se que o risco de COVID-19 (incidência de 12,6 casos/100 mil habitantes) na população piauiense seria 25 vezes maior que o risco de dengue (incidência de 0,5 casos/100 mil habitantes) na mesma população na SE 18.

Tal constatação reforça a necessidade de sensibilização dos profissionais para a suspeição e notificação dos casos. Além disso, apresenta-se o desafio de compreensão do desenvolvimento da epidemia "subdimensionada" de dengue simultaneamente à ocorrência de casos de COVID-19, em função dos sistemas de saúde mostrarem falhas assistenciais e certo desconhecimento a respeito dos efeitos da coinfecção em um mesmo indivíduo, o que pode resultar em sobrecarga ainda maior 6,14.

O enfretamento dessas doenças requer a implantação de estratégias eficazes de conscientização da população no sentido de eliminar criadouros de mosquitos, lavagem das mãos, uso de máscaras e, principalmente, distanciamento social. Além disso, é fundamental fortalecer o SUS e direcionar recursos para organizar os serviços de saúde, de forma a reduzir as iniquidades de acesso e oferecer a

\section{Figura 1}

Coeficiente de incidência de dengue e COVID-19. Piauí, Brasil, 2016-2020.

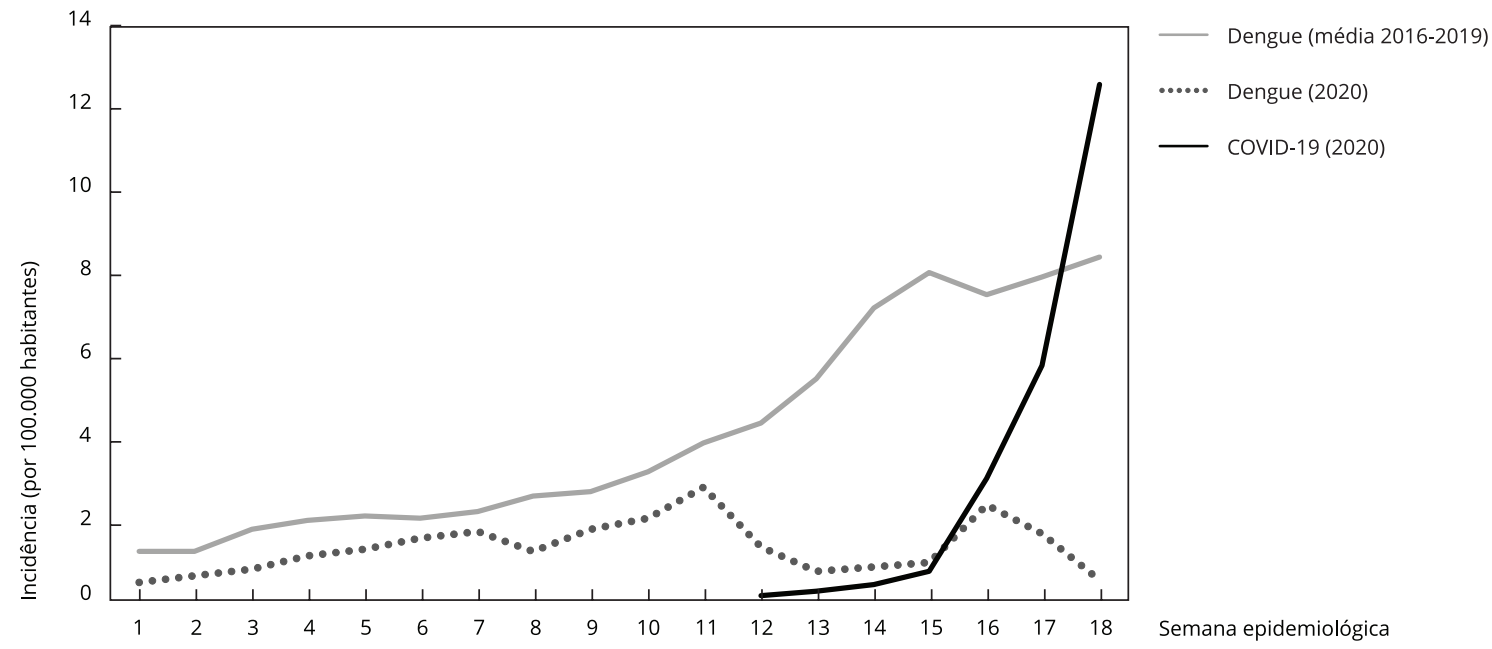


toda a população um atendimento de qualidade tanto na atenção primária à saúde, onde são atendidos os casos leves de ambas as doenças, como na assistência hospitalar direcionada aos casos de maior complexidade. Por ser concebida como acesso preferencial no atendimento de pessoas com suspeita de dengue e COVID-19, a atenção primária à saúde é um componente fundamental do sistema de saúde, devendo ser fortalecida e estar preparada para o atendimento oportuno, manejo clínico adequado e referenciamento dos casos graves, bem como atenção integral às condições sensíveis neste nível de atendimento 19 .

\section{Colaboradores}

M. D. M. Mascarenhas contribuiu com a concepção e projeto, análise e interpretação dos dados e redação do artigo; é responsável por todos os aspectos do trabalho na garantia da exatidão e integridade de qualquer parte da obra. F. M. A. Batista contribuiu com a concepção e projeto, análise e interpretação dos dados e redação do artigo; é responsável por todos os aspectos do trabalho na garantia da exatidão e integridade de qualquer parte da obra; e aprovou a versão final a ser publicada. M. T. P. Rodrigues, O. A. A. Barbosa e V. C. Barros contribuíram com a redação do artigo e revisão crítica relevante do conteúdo intelectual e aprovaram a versão final a ser publicada.

\section{Informações adicionais}

ORCID: Márcio Dênis Medeiros Mascarenhas (0000-0001-5064-2763); Francisca Miriane de Araújo Batista (0000-0002-0351-8994); Malvina Thais Pacheco Rodrigues (0000-0001-5501-0669); Ocimar de Alencar Alves Barbosa (0000-00015650-6877); Veruska Cavalcanti Barros (00000001-8483-4528).

\section{Conflitos de interesses}

Os autores declaram não haver conflitos de interesses.

\section{Referências}

1. World Health Organization. Coronavirus disease (COVID-19) outbreak. https://www.who. int/emergencies/diseases/novel-coronavi rus-2019 (acessado em 14/Mai/2020).

2. Williams G, Cañon-Montañez W. COVID-19: what we've learned so far. Rev Cuid (Bucaramanga) 2020; 11:e1225.

3. The Novel Coronavirus Pneumonia Emergency Response Epidemiology Team. The epidemiological characteristics of an outbreak of 2019 novel coronavirus diseases (COVID-19) - China, 2020. China CDC Weekly 2020; 2:113-22.

4. Walker PGT, Whittaker C, Watson O, Baguelin M, Ainslie KEC, Bhatia S, et al. The global impact of COVID-19 and strategies for mitigation and suppression. https://spiral.imperial. ac.uk:8443/handle/10044/1/77735 (acessado em 11/Mai/2020)

5. Barreto ML, Barros AJD, Carvalho MS, Codeço CT, Hallal PRC, Medronho RA. O que é urgente e necessário para subsidiar as políticas de enfrentamento da pandemia de COVID-19 no Brasil? Rev Bras Epidemiol 2020; 23:e200032.

6. Lorenz C, Azevedo TS, Chiaravalloti-Neto F. COVID-19 and dengue fever: a dangerous combination for the health system in Brazil. Travel Med Infect Dis 2020; [Epub ahead of print].

7. Monteiro ESC, Coelho ME, Cunha IS, Cavalcante MAS, Carvalho FAA. Aspectos epidemiológicos e vetoriais da dengue na cidade de Teresina, Piauí - Brasil, 2002 a 2006. Epidemiol Serv Saúde 2009; 18:365-74.

8. Croda J, Oliveira WK, Frutuoso RL, Mandetta LH, Baia-da-Silva DC, Brito-Sousa JD, et al. COVID-19 in Brazil: advantages of a socialized unified health system and preparation to contain cases. Rev Soc Bras Med Trop 2020; 53:e20200167.

9. Ferreira RCB, Papini S, Luchini LC, Vieira E. Persistence of malathion used in dengue control on household surfaces. Arq Inst Biol 2019; 86:e0042018. 
10. Barbosa IR, Tavares AM, Torres UPS, Nascimento CA, Moura MCBM, Vieira VB, et al. Identificação de áreas prioritárias para a vigilância e controle de dengue e outras arboviroses transmitidas pelo Aedes aegypti no Município de Natal - RN: relato de experiência. Epidemiol Serv Saúde 2017; 26:629-38.

11. Azevedo JVV, Santos CAC, Silva MT, Olinda RA, Santos DAS. Análise das variações climáticas na ocorrência de doenças respiratórias por influenza em idosos na região metropolitana de João Pessoa - PB. Sociedade \& Natureza 2017; 29:123-35.

12. Oliveira WK, Duarte E, França GVA, Garcia LP. How Brazil can hold back COVID-19. Epidemiol Serv Saúde 2020; 29:e2020044.

13. Moreira RS. COVID-19: unidades de terapia intensiva, ventiladores mecânicos e perfis latentes de mortalidade associados à letalidade no Brasil. Cad Saúde Pública 2020; 36:e00080020.

14. Yan G, Lee CK, Lam LTM, Yan B, Chua YX, Lim AYN, et al. Covert COVID-19 and falsepositive dengue serology in Singapore. Lancet Infect Dis 2020; 20:536.

15. Velasco MS, Chilet CC, Rodriguez RP, Urbina $A G$, Berrospi FI. Coinfección entre dengue y COVID-19: necesidad de abordaje en zonas endémicas. Rev Fac Cienc Méd (Córdoba) 2020; 77:52-4.
16. Ministério da Saúde. Monitoramento dos casos de arboviroses urbanas transmitidas pelo Aedes aegypti (dengue, chikungunya e zika), Semanas Epidemiológicas 1 a 17, 2020. Bol Epidemiol 2020; 51(18). https://www.saude.gov.br/ima ges/pdf/2020/May/04/Boletim-epidemiologi co-SVS-18.pdf.

17. Secretaria de Saúde do Estado do Piauí. Dengue, chikungunya, zika e microcefalia. Boletim da 17a Semana Epidemiológica 2020. http:// www.saude.pi.gov.br/uploads/warning_docu ment/file/530/Boletim_Epidemiol\%C3\%B3gi co_PI_SE_17\%C2\%AA_2020.pdf.

18. Secretaria de Saúde do Estado do Piauí. Painel epidemiológico COVID-19 Piauí. https:// datastudio.google.com/reporting/a6dc07e94161-4b5a-9f2a-6f9be486e8f9/page/2itOB (acessado em 11/Mai/2020).

19. Souza CDF, Gois-Santos VT, Correia DS, Martins-Filho PR, Santos VS. The need to strengthen primary health care in Brazil in the context of the COVID-19 pandemic. Braz Oral Res 2020; 34:e047.
Recebido em 15/Mai/2020

Versão final reapresentada em 20/Mai/2020

Aprovado em 21/Mai/2020 